advanced cancer diagnosis with a $37 \%$ reduction in endoscopy activity in County Durham, we were unable to show a significant increase in short term cancer related mortality. We believe a similar phenomenon is occurring across the NHS leading to an indirect increase in COVID-19 related cancer diagnosis. Measures to mitigate this are urgently needed.

\section{PTH-21 CAPNOGRAPHY MONITORING OF PHYSICIAN-LED PROCEDURAL SEDATION FOR GASTROINTESTINAL SERVICES AT A UK TEACHING HOSPITAL}

${ }^{1}$ Gareth Corbett, 'Peter Pugh, 'Jurgen Herre, ${ }^{1}$ Teik Choon See, 'David de MonteverdeRobb, ${ }^{2}$ Rafael Torrejon Torres*, ${ }^{2}$ Rhodri Saunders, ${ }^{3}$ Catherine Leonard, ${ }^{1}$ Amit Prakash. ${ }^{1}$ Cambridge University Hospital NHS Trust, Cambridge, UK; ${ }^{2}$ Coreva Scientific, Koenigswinter, Germany; ${ }^{3}$ Medtronic UK, Watford, UK

\subsection{6/gutjpl-2021-BSG.336}

Introduction Physician-led sedation is commonly employed for selected endoscopic procedures. There is still an open question around the optimal patient monitoring strategy during such procedures. Use of additional capnography has been shown to be associated with fewer patient respiratory compromise events than pulse oximetry alone. To quantify and explore the impact of capnography on patient safety, a quality improvement initiative was undertaken for gastrointestinal (GI), interventional cardiology (IC), vascular access (VA), and respiratory medicine (RM) clinical services at a large UK teaching hospital.

Methods Four target events as defined by the world Society for Intravenous Anaesthesia (SIVA) tool were determined as the cumulative primary outcome after a review of available literature. These events were: Oxygen desaturation $(75-90 \%)$ for $<60$ s, severe $(<75 \%$ at any time) or prolonged $(<90 \%$ for $>60$ s) oxygen desaturation, bradycardia ( $>25 \%$ increase from baseline), and tachycardia ( $>25 \%$ decrease from baseline). The design was pre- and post-implementation of capnography monitoring. A $20 \%$ reduction in this cumulative endpoint was agreed upon to be the quality improvement threshold. Data on procedures featuring procedural sedation were collected as a convenience sample between December 2017 and January 2020. The results were entered on-site in an Excel based data collection tool. No patient identifiers were recorded.

Results The data from 1,401 procedures across the GI, IC, and RM services were collected. The first 666 procedures were pre-capnography (baseline), with the subsequential 735 post-capnography implementation (capnography). GI represented 601 of the procedures, with 262 collected at baseline and 339 with capnography. Over the 1,401 procedures, a $42 \%$ reduction in the incidence of the composite endpoint was recorded. The adjusted odds ratio was estimated at 0.57 (95\% confidence interval (CI): 0.42 - 0.77).

For the department of GI, 20 events were observed in the baseline procedures (0.076 events per procedure), and for the capnography arm 12 (0.035 events per procedure), corresponding with a $53.9 \%$ percent reduction in the composite endpoint. Odds ratios were decreased for all American Society of Anesthesiologists (ASA) levels, with ASA III patients receiving capnography being associated with the lowest odds ratio [0.24 (95\% CI: $0.06-0.94)]$ for the composite primary outcome compared to baseline.

Conclusions Implementing capnography monitoring led to a $42 \%$ overall and a $53.9 \%$ GI-specific reduction in the composite outcome patient safety events. Participating services support capnography monitoring being added to the hospital's sedation guidelines. More data are required to explore whether reduction in more rare but severe patient outcomes can be realised with use of capnography monitoring.

\section{PTH-22 BENCHMARKING IBD PHARMACY SERVICES TO OPTIMISE, STRENGTHEN AND ALIGN IBD EXPERT PHARMACY PRACTICE}

\author{
${ }^{1,2}$ Anja St* ${ }^{*}, 3$ Uchu Meade. 'Brighton and Sussex University Hospitals NHS Trust, Brighton, \\ UK; ${ }^{2}$ IBDUK, Hatfield, UK; ${ }^{3}$ St Mark's Hospital, Harrow, UK
}

\subsection{6/gutjnl-2021-BSG.337}

Introduction UK IBD Standards 2019 (ibduk.org) for the first time embed and describe Specialist Pharmacy Services (SPS) as an integral part of the IBD multidisciplinary team (MDT) and enable recognition and commissioning of expert pharmacy practice $^{1}$. The IBD UK National Service Standards Audit 2020 provides a benchmark of IBD pharmacy standards.

Methods The benchmarking tool developed and agreed by IBD UK to drive quality defines A-D descriptors for all standards ( $\mathrm{A}=$ 'excellent, proactive' to $\mathrm{D}=$ 'minimal, inadequate' care). Descriptors for the $4(7 \%)$ IBD Standards describing SPS were developed in 2 consensus workshops by IBD UK with expert pharmacy representation. Royal Pharmaceutical Society (RPS) standards and SPS feedback were used to define descriptors for pharmacy leadership, medicines expert role and MDT working. All UK IBD services were asked to complete the self-assessment between Oct 2019 and Jan 2020.

Results 166 (72\%) paediatric and adult IBD services took part across the UK.

MDT Standard $46 \% \quad(n=81 / 166)$ of all IBD Services have pharmacist input to the IBD MDT, but only $27 \%(n=18 / 66)$ of adult IBD Services met or exceeded the IBD Standard of 0.6 WTE Expert Pharmacist in IBD (EPharmIBD)/250,000 population.

Leadership role $76 \%(n=98 / 129)$ of services with an IBD leadership team work with a pharmacist of which $48 \%(n=47 / 98)$ work with an EPharmIBD on the annual formulary review and of these teams $66 \%(n=31 / 47)$ work with an EPharmIBD on annual protocol/policy review, with actions and outcomes, to actively develop pharmacy services within IBD.

Medicines expert role Ward pharmacists in 95\% $(n=157 / 166)$ of IBD Services have access to an advanced generalist pharmacist for advice and of these services 54\% ( $n=84 / 157)$ are supported by an EPharmIBD but in only 41\% $(n=34 / 84)$ of these services patients and ward pharmacists have access to an EPharmIBD on admission and during their stay for medication review, optimisation and personalised consultation.

Conclusions Benchmarking shows a low level of pharmacy IBD expertise in the UK with a minority of services, ward pharmacists and patients having access to an EPharmIBD. Less than half of IBD leadership teams work with an EPharmIBD and a small number of services have adequate WTE of EPharmIBDs commissioned.

With new treatments becoming available the role will become more essential. The benchmarking tool highlights the high need for EPharmIBDs, advanced competencies in pharmacy IBD practice, training pathways to develop future EPharmIBDs and prioritisation of service commissioning. The pharmacy profession needs to respond to this challenge urgently to ensure high quality pharmaceutical care for IBD patients. 


\section{REFERENCE}

1. St.Clair Jones A, Meade U. UK IBD Standards: a roadmap to IBD pharmacy workforce transformation. BSG Campus 2021, Poster 223

\section{PTH-23 CONSULTANT TRIAGE OF ENDOSCOPY WAITING LISTS DURING THE COVID-19 PANDEMIC SAVES MONEY AND REDUCES WORKLOAD}

Rachel Edwards*, Peter Foulser, Stuart Gould, Aik Lim. Epsom and St Helier University Hospitals NHS Trust, UK

\subsection{6/gutjnl-2021-BSG.338}

Introduction The Covid-19 pandemic continues to cause delays nationally in provision of endoscopy services. As recommended by the BSG, triage of 'Planned' and 'Routine' endoscopy waiting lists was conducted within a hospital serving a population of 166,257 .

This study aimed to see if consultant led triage resulted in cost savings and/or a reduction in endoscopy workload.

Methods The 'Planned' endoscopy waiting list included patients on a surveillance pathway or awaiting a follow up procedure. The 'Routine' endoscopy waiting list referred to all other outpatient endoscopy requests. Patient records were reviewed on iSOFT Clinical Manager 2.0. Patients were telephoned if further information was needed.

Each endoscopy request was assigned to one of four categories; 'no longer required', 'no change to request', 'upgrade request to urgent', and 'change request to alternative planned interval'. Outcomes were recorded in an Excel spreadsheet. Patients were informed of any important change in management plan.

Procedures that were deemed no longer required were reviewed in more detail to establish the reason for this. Potential savings made as a result of not carrying out procedures that were categorised as 'no longer required' were based on the 2018/19 National Tariff for the procedure with biopsies.

Results A total of 589 patients were on the endoscopy waiting lists; 326 on the 'Planned' list, and 263 on the 'Routine' list.

$157(27 \%)$ of endoscopy requests were categorised as not required. Reasons for this included updated or incorrect adherence to surveillance guidelines (26\%), clinical judgement deeming it no longer necessary (62\%), or a change in the patient's clinical status or unclear indication (12\%).

Conclusions $1.27 \%$ of endoscopy waiting list procedures were judged unnecessary.

- Consultant led triage of endoscopy waiting lists resulted in an estimated cost saving of $£ 67,993$ (a $25 \%$ cost reduction) and a reduction in endoscopy work load.

- Potential savings nationally from endoscopy waiting list triage are considerable.

Abstract PTH-23 Table 1 Numbers of endoscopy requests triaged into each category across planned and routine waiting lists

\begin{tabular}{lll}
\hline $\begin{array}{l}\text { Outcome after consultant triage of endoscopy } \\
\text { request }\end{array}$ & Planned & Routine \\
\hline No longer required & 79 & 78 \\
No change to request & 202 & 159 \\
Upgrade request to urgent & 14 & 9 \\
Change request to alternative planned interval & 31 & 17 \\
Total & 326 & 263 \\
\hline
\end{tabular}

- Triage theoretically increased training opportunities by reducing workload.

Greater awareness of current guidelines could reduce the number of endoscopies scheduled.

\section{PTH-24 THE IMPACT OF PEG COMPLICATIONS PRESENTING TO ED - A DGH EXPERIENCE}

Sanjay Gupta*. Croydon University Hospital, Croydon, Surrey, UK

\subsection{6/gutjnl-2021-BSG.339}

Introduction Percutaneous endoscopic gastrostomy (PEG) tube and Radiologically inserted percutaneous gastrostomy (RIG) tube feeding is widely used for providing enteral nutrition to patients with impaired swallowing or cannot meet their nutritional requirements by oral route. At a district general hospital (DGH), patients present with complications of gastrostomy tubes to the emergency department (ED). I analysed the data to determine the impact of these patients on acute services including length of stay (LOS) in hospital and associated costs. Methods A list of patients who attended ED with gastrostomy related problems from April 2018 to August 2019 was obtained and analysed. Indication for referrals, length of stay in hospital and outcomes with regards to the feeding tubes were analysed. Cost analysis was conducted using the 2017/18 NHS tariffs.

Results Based on coding, 80 patients were identified of which 3 were paediatric patients, 1 had nasojejunal tube problems, another's details could not be verified and 1 had an unrelated admission - these were excluded from analysis. Of the 74 patients, there were 45 attendances in the first year and 29 in the 5 months from April 2019. Mean age was 62 years (2284). The reasons for presentation included dislodged tubes $(\mathrm{n}=38,51 \%)$, blocked tubes $(\mathrm{n}=16,22 \%)$, to check PEG position replaced in community/sent with a deflated balloon $(n=6)$, infection/pain/bleeding at insertion site $(n=7)$, split tubes $(n=2)$, clamp problems $(n=1)$ and suspected buried bumper $(\mathrm{n}=3)$.

LOS varied from 2 to 47 days $(n=39)$ with a mean of 9.3 days and a cumulative total of 353 bed days. 35 patients $(47 \%)$ were managed in ED, $13(18 \%)$ in 2 days, 1 in 3 days, 4 on day 4 while 22 patients (30\%) stayed beyond 5 days. 2 patients died during hospital stay. 14 patients (19\%) attended more than once, with 2 patients attending 6 and 7 times respectively. Most of the blocked and dislodged feeding tubes were replaced by ED doctors or the gastroenterology team. 12 patients had gastrostomy tubes insertion by Interventional radiology (usually with tract dilatation) with a mean delay of 6.6 days (1-31). 2 patients had endoscopic gastrostomy insertion on day 9 and 11 after admission. With an estimate of $£ 160$ for $\mathrm{ED}$ attendance and $£ 346$ for each bed day, the cost for managing patients with PEG complications was $£ 133,978$.

Conclusions Care of gastrostomy tubes in the community needs improvement to avoid distress to patients/carers and reduce pressure on acute services as well as prevent repeat attendances. This requires greater support, expertise and training of community healthcare professionals. A focused outreach gastroenterology specialist advice service can help in reducing ED attendances, thus saving bed days and reducing costs to the NHS. 